\title{
Performance evaluation of two fuzzy-based cluster head selection systems for wireless sensor networks
}

\author{
Junpei Anno ${ }^{\mathrm{a}}$, Leonard Barolli ${ }^{\mathrm{b}, *}$, Arjan Durresi ${ }^{\mathrm{c}}$, Fatos Xhafa ${ }^{\mathrm{d}}$ and Akio Koyama ${ }^{\mathrm{e}}$ \\ ${ }^{a}$ Graduate School of Engineering, Fukuoka Institute of Technology (FIT), Higashi-Ku, Fukuoka, Japan \\ ${ }^{\mathrm{b}}$ Department of Information and Communication Engineering, Fukuoka Institute of Technology (FIT), \\ Higashi-Ku, Fukuoka, Japan \\ ${ }^{\mathrm{c}}$ Department of Computer and Information Science, Indiana University Purdue University at \\ Indianapolis, Indianapolis, IN, USA \\ ${ }^{\mathrm{d}}$ Department of Languages and Informatics Systems, Polytechnic University of Catalonia, Barcelona, \\ Spain \\ ${ }^{\mathrm{e}}$ Department of Informatics, Yamagata University, Yonezawa, Yamagata, Japan
}

\begin{abstract}
Sensor networks supported by recent technological advances in low power wireless communications along with silicon integration of various functionalities are emerging as a critically important computer class that enable novel and low cost applications. There are many fundamental problems that sensor networks research will have to address in order to ensure a reasonable degree of cost and system quality. Cluster formation and cluster head selection are important problems in sensor network applications and can drastically affect the network's communication energy dissipation. However, selecting of the cluster head is not easy in different environments which may have different characteristics. In this paper, in order to deal with this problem, we propose two fuzzy-based systems for cluster head selection in sensor networks. We call these systems: FCHS System 1 and FCHS System2. We evaluate the proposed systems by simulations and have shown that FCHS System 2 make a good selection of the cluster head compared with FCHS System1 and another previous system.
\end{abstract}

\section{Introduction}

Recent developments in technologies such as wireless communication and microelectronics have enabled Wireless Sensor Network (WSN) applications to be deployed for many applications such as battlefield surveillance and environment monitoring. An important aspect of such networks is that the nodes are unattended, resource-constrained, their energy cannot be replenished and network topology is unknown. The resource-constrained limitations make it essential for these sensor nodes to conserve energy to increase life-time of the sensor network $[1-3,15,16]$.

Recently, there are lot of research efforts towards the optimization of standard communication paradigms for such networks. In fact, the traditional Wireless Network (WN) design has never paid attention to constraints such as the limited or scarce energy of nodes and their computational power.

\footnotetext{
${ }^{*}$ Corresponding author: Leonard Barolli, Department of Information and Communication Engineering, Fukuoka Institute of Technology (FIT), 3-30-1 Wajiro-Higashi, Higashi-Ku, Fukuoka 811-0295, Japan. E-mail: barolli@ fit.ac.jp.
} 
Also, in WSN paths can change over time, because of time-varying characteristics of links, local contention level and nodes reliability. These problems are important especially in a multi-hop scenario, where nodes accomplish also at the routing of other nodes' packets [3,22].

There are many fundamental problems that sensor networks research will have to address in order to ensure a reasonable degree of cost and system quality. Some of these problems include sensor node clustering, Cluster Head $(\mathrm{CH})$ selection and energy dissipation. There are many research works that deal with these challenges [4,9-13,17-19].

The cluster based algorithms could be used for partitioning the sensor nodes into subgroups for task subdivision or energy management. Cluster formation is one of most important problems in sensor network applications and can drastically affect the network's communication energy dissipation. Clustering is performed by assigning each sensor node to a specific $\mathrm{CH}$. All communication to (from) each sensor node is carried out through its corresponding $\mathrm{CH}$ node. Obviously one would like to have each sensor to communicate with the closest $\mathrm{CH}$ node to conserve its energy, however $\mathrm{CH}$ nodes can usually handle a specific number of communication channels. Therefore, there is a maximum number of sensors that each $\mathrm{CH}$ node can handle. This does not allow each sensor to communicate to its closest $\mathrm{CH}$ node, because the $\mathrm{CH}$ node might have already reached its service capacity. $\mathrm{CHs}$ can fuse data from sensors to minimize the amount of data to be sent to the sink. When network size increases, clusters can also be organized hierarchically.

In the conventional cluster architecture, clusters are formed statically at the time of network deployment. The attributes of each cluster, such as the size of a cluster, the area it covers, and the members it possesses, are static. In spite of its simplicity, the static cluster architecture suffers from several drawbacks. The fixed membership is not robust from the perspective of fault tolerance. If a $\mathrm{CH}$ dies of power depletion, all the sensors in the cluster render useless. Also, fixed membership prevents sensor nodes in different clusters from sharing information and collaborating on data processing. Dynamic cluster architectures, on the other hand, offer several desirable features. Formation of a cluster is triggered by certain events of interest.

In this work, we deal with the $\mathrm{CH}$ selection. A method for $\mathrm{CH}$ selection is as follows. When a sensor with sufficient battery and computational power detects (with a high Signal-to-Noise Ratio: SNR) signals of interest, it volunteers to act as a $\mathrm{CH}$. This is a simple method, because no explicit leader $(\mathrm{CH})$ election is required and, hence, no excessive message exchanges are incurred. However, selecting of the $\mathrm{CH}$ in this way is not easy in different environments which may have different characteristics such as error rate, SNR, throughput and so on.

The heuristic approaches based on Fuzzy Logic (FL) and Genetic Algorithms (GA) can prove to be efficient for traffic control in wireless networks $[6-8,14]$.

In this paper, in order to deal with this problem, we propose two fuzzy-based systems for cluster head selection in sensor networks. We call these systems: FCHS System1 and FCHS System2. We evaluate the proposed systems by simulations and have shown that FCHS System 2 make a good selection of the cluster head compared with FCHS System1 and another previous system.

The paper is organized as follows. In Section 2, we discuss the related work. In Section 3, we introduce the proposed systems design. In Section 4, we present the simulation results. Conclusions are given in Section 5.

\section{Related work}

In this section, we review related work in clustering algorithms. Several clustering methods such as weighted clustering [11], hierarchal clustering [9] and dynamic clustering [12] algorithms have been 


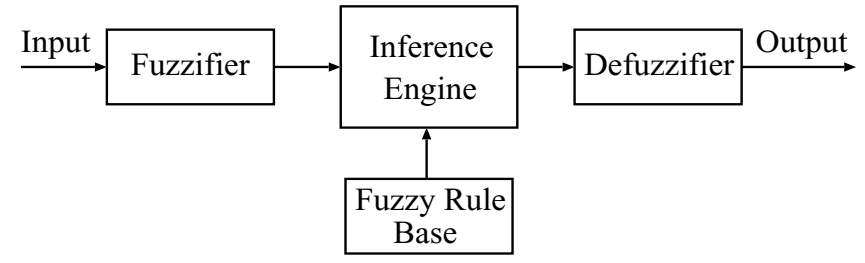

Fig. 1. FLC structure.

proposed to organize nodes as a cluster. Most algorithms elect leaders based on certain weights or iteratively optimize a cost function or use heuristic to generate minimum number of clusters. The Distributed Clustering Algorithm (DCA) [10] assumes quasi-stationary nodes with real-valued weights. The Weighted Clustering Algorithm [11] elects a node based on the number of neighbors, transmission power and so on. The Max-Min d-Clustering Algorithm [4] generates d-hop clusters with a run time of $\mathrm{O}(\mathrm{d})$ rounds. This algorithm does not minimize the communicating complexity of sending information to the information center.

The hierarchal clustering scheme [9] uses spanning tree-based approach to produce cluster with certain properties. However, energy efficiency is not addressed in this work. In [13], the authors have proposed an emergent algorithm that iteratively tries to achieve high packing efficiency, however negotiation among nodes to be $\mathrm{CH}$ and join cluster based on degree and proximity leads to high amount of communication overhead, thus wastage energy.

LEACH $[17,18]$ uses two-layered architecture for data dissemination. In this scheme, sensors periodically elect themselves as $\mathrm{CHs}$ with some probability and broadcast an invitation message for nearby nodes to join the cluster. The nodes that do not intend to be $\mathrm{CHs}$ join the cluster based on the proximity of $\mathrm{CH}$, thus minimizing the communicating cost. However, LEACH and PEGASIS [19] require the apriory knowledge of the network topology.

In [20], the authors propose a self-reconfiguring protocol for Wireless Personal Area Networks (WPAN) using an unsupervised clustering method. A fuzzy logic system is used to select the master/controller for each cluster. In our previous work [5], we had shown by simulation results that the selection surface of our system was better than the system in [20].

\section{Proposed systems}

In this section, we present the design of Fuzzy-based CH Selection (FCHS) systems.

\subsection{FLC structure}

The Fuzzy Logic Controller (FLC) basic elements are shown in Fig. 1. They are the fuzzifier, inference engine, Fuzzy Rule Base (FRB) and defuzzifier. As shown in Fig. 2, as membership functions we use triangular and trapezoidal membership functions because they are suitable for real-time operation [21]. They are shown in Fig. 2 and are given as:

$$
f\left(x ; x_{0}, a_{0}, a_{1}\right)= \begin{cases}\frac{x-x_{0}}{a_{0}}+1 & \text { for } x_{0}-a_{0}<x \leqslant x_{0} \\ \frac{x_{0}-x}{a_{1}}+1 & \text { for } x_{0}<x \leqslant x_{0}+a_{1} \\ 0 & \text { otherwise }\end{cases}
$$




\begin{tabular}{ccccc}
\multicolumn{5}{c}{ Table 1 } \\
\multicolumn{7}{c}{ FRB1 } & \\
\hline Rule & DS & SP & NT & PCHS \\
\hline 1 & NR & LO & LI & LS \\
2 & NR & LO & MU & MD \\
3 & NR & LO & HE & LW \\
4 & NR & ME & LI & S \\
5 & NR & ME & MU & LS \\
6 & NR & ME & HE & MD \\
7 & NR & HG & LI & VS \\
8 & NR & HG & MU & S \\
9 & NR & HG & HE & LS \\
10 & MO & LO & LI & MD \\
11 & MO & LO & MU & LW \\
12 & MO & LO & HE & W \\
13 & MO & ME & LI & LS \\
14 & MO & ME & MU & MD \\
15 & MO & ME & HE & LW \\
16 & MO & HG & LI & S \\
17 & MO & HG & MU & LS \\
18 & MO & HG & HE & MD \\
19 & FA & LO & LI & LW \\
20 & FA & LO & MU & W \\
21 & FA & LO & HE & VW \\
22 & FA & ME & LI & MD \\
23 & FA & ME & MU & LW \\
24 & FA & ME & HE & W \\
25 & FA & HG & LI & LS \\
26 & FA & HG & MU & MD \\
27 & FA & HG & HE & LW \\
\hline
\end{tabular}
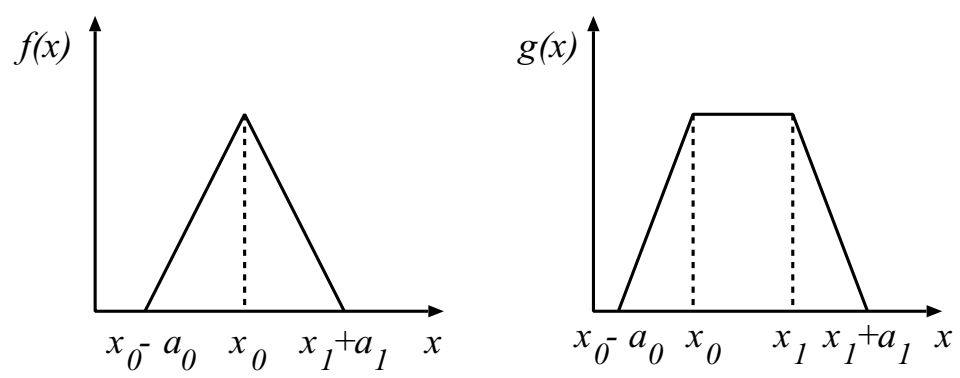

Fig. 2. Triangular and trapezoidal membership functions.

$$
g\left(x ; x_{0}, x_{1}, a_{0}, a_{1}\right)= \begin{cases}\frac{x-x_{0}}{a_{0}}+1 & \text { for } x_{0}-a_{0}<x \leqslant x_{0} \\ 1 & \text { for } x_{0}<x \leqslant x_{1} \\ \frac{x_{1}-x}{a_{1}}+1 & \text { for } x_{1}<x \leqslant x_{1}+a_{1} \\ 0 & \text { otherwise }\end{cases}
$$

where $x_{0}$ in $f($.$) is the center of triangular function; x_{0}\left(x_{1}\right)$ in $g($.$) is the left (right) edge of trapezoidal$ function; and $a_{0}\left(a_{1}\right)$ is the left (right) width of the triangular or trapezoidal function.

\subsection{Design of FCHS system1}

In FCHS System1, the input linguistic parameters are: 
- Distance of Cluster Centroid (DS);

- Remaining Battery Power of Sensor (SP);

- Network Traffic (NT).

The term sets for each input linguistic parameter are defined respectively as:

$$
\begin{aligned}
T(D S) & =\{\text { Near }(N R), \text { Moderate }(M O), \text { Far }(F A)\} ; \\
T(S P) & =\{\text { Low }(\text { LO }), \text { Moderate }(M E), \text { High }(H G)\} ; \\
T(N T) & =\{\text { Light }(\text { LI }), \text { Moderate }(M U), \text { Heavy }(H E)\} .
\end{aligned}
$$

The membership functions for input parameters of FLC1 are defined as follows:

$$
\begin{aligned}
\mu_{N R}(D S) & =g\left(D S ; N R_{0}, N R_{1}, N R_{w 0}, N R_{w 1}\right) ; \\
\mu_{M O}(D S) & =f\left(D S ; M O_{0}, M O_{w 0}, M O_{w 1}\right) ; \\
\mu_{F A}(D S) & =g\left(D S ; F A_{0}, F A_{1}, F A_{w 0}, F A_{w 1}\right) ; \\
\mu_{L O}(S P) & =g\left(S P ; L O_{0}, L O_{1}, L O_{w 0}, L O_{w 1}\right) ; \\
\mu_{M E}(S P) & =g\left(S P ; M E_{0}, M E_{1}, M E_{w 0}, M E_{w 1}\right) ; \\
\mu_{H G}(S P) & =g\left(S P ; H G_{0}, H G_{1}, H G_{w 0}, H G_{w 1}\right) ; \\
\mu_{L I}(N T) & =g\left(N T ; L I_{0}, L I_{1}, L I_{w 0}, L I_{w 1}\right) ; \\
\mu_{M U}(N T) & =f\left(N T ; M U_{0}, M U_{w 0}, M U_{w 1}\right) ; \\
\mu_{H E}(N T) & =g\left(N T ; H E_{0}, H E_{1}, H E_{w 0}, H E_{w 1}\right) .
\end{aligned}
$$

The small letters $w 0$ and $w 1$ mean left width and right width, respectively.

The output linguistic parameter is the Possibility of $\mathrm{CH}$ Selection. We write for short as PCHS. We define the term set of PCHS as: $\{\operatorname{Very} W e a k(V W)$, Weak $(W)$, Little Weak $(L W)$, Medium (MD), Little Strong (LS), Strong $(S)$, Very Strong $(V S)\}$.

The membership functions for the output parameter PCHS are defined as follows:

$$
\begin{aligned}
\mu_{V W}(P C H S) & =g\left(P C H S ; V W_{0}, V W_{1}, V W_{w 0}, V W_{w 1}\right) ; \\
\mu_{W}(P C H S) & =f\left(P C H S ; W_{0}, W_{w 0}, W_{w 1}\right) ; \\
\mu_{L W}(P C H S) & =f\left(P C H S ; L W_{0}, L W_{w 0}, L W_{w 1}\right) ; \\
\mu_{M D}(P C H S) & =f\left(P C H S ; M D_{0}, M D_{w 0}, M D_{w 1}\right) ; \\
\mu_{L S}(P C H S) & =f\left(P C H S ; L S_{0}, L S_{w 0}, L S_{w 1}\right) ; \\
\mu_{S}(P C H S) & =f\left(P C H S ; S_{0}, S_{w 0}, S_{w 1}\right) ; \\
\mu_{V S}(P C H S) & =g\left(P C H S ; V S_{0}, V S_{1}, V S_{w 0}, V S_{w 1}\right) .
\end{aligned}
$$

The fuzzy membership functions for input parameters are shown in Fig. 3. The FRB is shown in Table 2 and forms a fuzzy set of dimensions $|T(D S)| \times|T(S P)| \times|T(N T)|$, where $|T(x)|$ is the number of terms on $T(x)$. The FRB1 has 27 rules. The control rules have the following form: IF "conditions" THEN "control action". 


\begin{tabular}{|c|c|c|c|c|}
\hline Rule & RPS & D3N & DCC & PCHS \\
\hline 1 & $\overline{\mathrm{Lo}}$ & $\overline{F W}$ & $\mathrm{Nr}$ & VW \\
\hline 2 & Lo & $\mathrm{FW}$ & Мo & W \\
\hline 3 & Lo & FW & $\mathrm{Fr}$ & W \\
\hline 4 & Lo & $\mathrm{Me}$ & $\mathrm{Nr}$ & W \\
\hline 5 & Lo & $\mathrm{Me}$ & Mo & W \\
\hline 6 & Lo & $\mathrm{Me}$ & $\mathrm{Fr}$ & W \\
\hline 7 & Lo & $\mathrm{Mn}$ & $\mathrm{Nr}$ & VW \\
\hline 8 & Lo & $\mathrm{Mn}$ & Мо & VW \\
\hline 9 & Lo & $\mathrm{Mn}$ & $\mathrm{Fr}$ & VW \\
\hline 10 & $\mathrm{Mi}$ & Fw & $\mathrm{Nr}$ & W \\
\hline 11 & $\mathrm{Mi}$ & $\mathrm{FW}$ & Mo & LW \\
\hline 12 & $\mathrm{Mi}$ & $\mathrm{Fw}$ & $\mathrm{Fr}$ & MD \\
\hline 13 & $\mathrm{Mi}$ & $\mathrm{Me}$ & $\mathrm{Nr}$ & LW \\
\hline 14 & $\mathrm{Mi}$ & $\mathrm{Me}$ & Мо & MD \\
\hline 15 & Mi & $\mathrm{Me}$ & Fr & LS \\
\hline 16 & $\mathrm{Mi}$ & $\mathrm{Mn}$ & $\mathrm{Nr}$ & MD \\
\hline 17 & $\mathrm{Mi}$ & $\mathrm{Mn}$ & Мо & LS \\
\hline 18 & $\mathrm{Mi}$ & $\mathrm{Mn}$ & $\mathrm{Fr}$ & $S$ \\
\hline 19 & $\mathrm{Hg}$ & $\mathrm{Fw}$ & $\mathrm{Nr}$ & LW \\
\hline 20 & $\mathrm{Hg}$ & $\mathrm{FW}$ & Мо & MD \\
\hline 21 & $\mathrm{Hg}$ & Fw & Fr & LS \\
\hline 22 & $\mathrm{Hg}$ & $\mathrm{Me}$ & $\mathrm{Nr}$ & MD \\
\hline 23 & $\mathrm{Hg}$ & $\mathrm{Me}$ & Мо & LS \\
\hline 24 & $\mathrm{Hg}$ & $\mathrm{Me}$ & $\mathrm{Fr}$ & $S$ \\
\hline 25 & $\mathrm{Hg}$ & $\mathrm{Mn}$ & $\mathrm{Nr}$ & LS \\
\hline 26 & $\mathrm{Hg}$ & $\mathrm{Mn}$ & Мо & S \\
\hline 27 & $\mathrm{Hg}$ & $\mathrm{Mn}$ & $\mathrm{Fr}$ & VS \\
\hline
\end{tabular}

\subsection{Design of FCHS system2}

We found that the number of the neighbor nodes is very important for the selection of the $\mathrm{CH}$. To explain this effect let us consider a small network model with 14 nodes as shown in Fig. 4. In this figure, the node number 1 has 6 neighbor nodes, for this reason is selected as a $\mathrm{CH}$. After that, 2 other sets of nodes remain, but node 2 has more neighbor nodes than nodes 3 and 7 , thus node 2 is selected as $\mathrm{CH}$. Finally, if we see 2 other remained nodes (node 3 and node 7), they have the same neighbor nodes that are not included in other clusters. In this case, the node with higher ID number is selected as CH. For this reason, node 7 is selected as $\mathrm{CH}$ and the procedure of $\mathrm{CH}$ selection is finished as shown in Fig. 5.

By considering the number of neighbor nodes as a fuzzy input parameter, we propose 3 input linguistic parameters for FCHS System2:

- Remaining Battery Power of Sensor (RPS);

- Degree of Number of Neighbor Nodes (D3N);

- Distance from Cluster Centroid (DCC).

The term sets for each input linguistic parameters are defined respectively as:

$$
\begin{aligned}
T(R P S) & =\{\operatorname{Low}(\operatorname{Lo}), \text { Middle }(M i), \operatorname{High}(H g)\} ; \\
T(D 3 N) & =\{F e w(F w), \text { Medium }(M e), \operatorname{Many}(M n)\} ; \\
T(D C C) & =\{\operatorname{Near}(N r), \text { Moderate }(M o), \operatorname{Far}(F r)\} .
\end{aligned}
$$



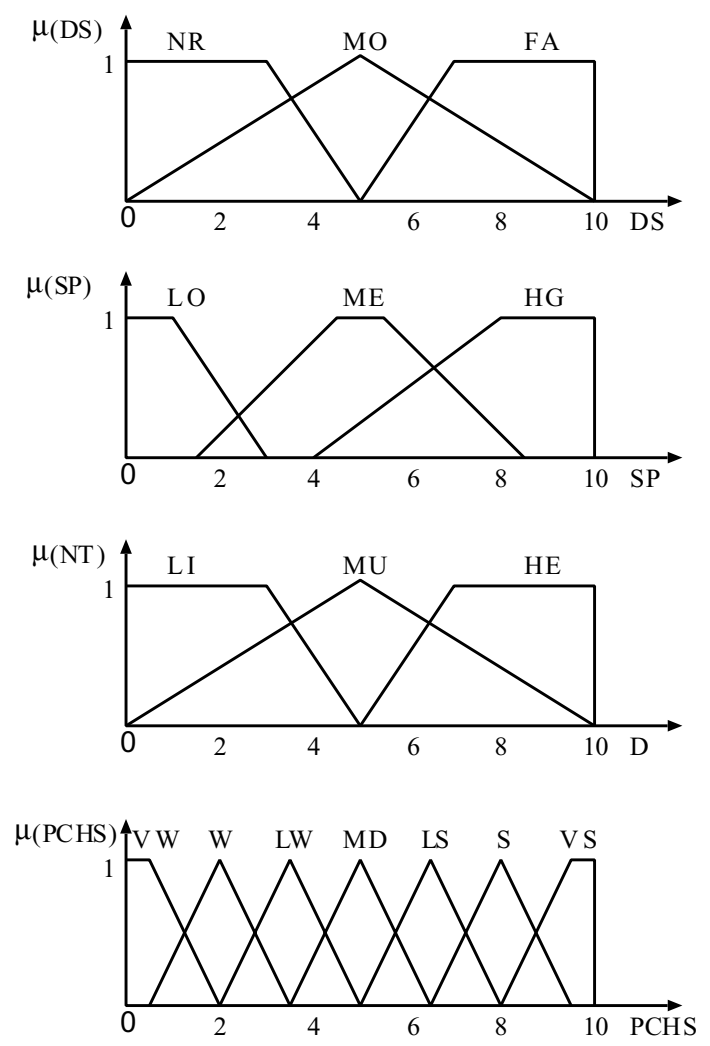

Fig. 3. Membership functions for FCHS system1.

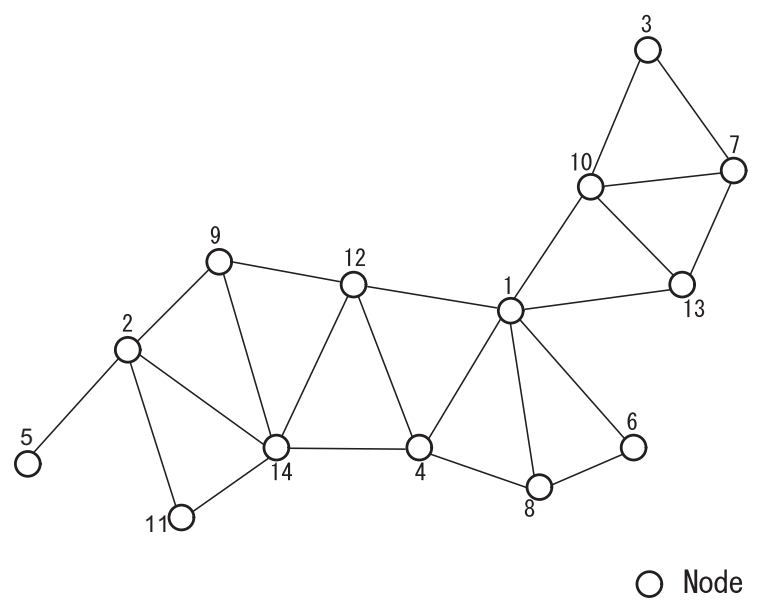

Fig. 4. A network model with 14 nodes.

The membership functions for input linguistic parameters of FLC are defined as follows:

$$
\begin{aligned}
\mu_{L o}(R P S) & =g\left(R P S ; L o_{0}, L o_{1}, L o_{w 0}, L o_{w 1}\right) ; \\
\mu_{M i}(R P S) & =f\left(R P S ; M i_{0}, M i_{w 0}, M i_{w 1}\right) ;
\end{aligned}
$$




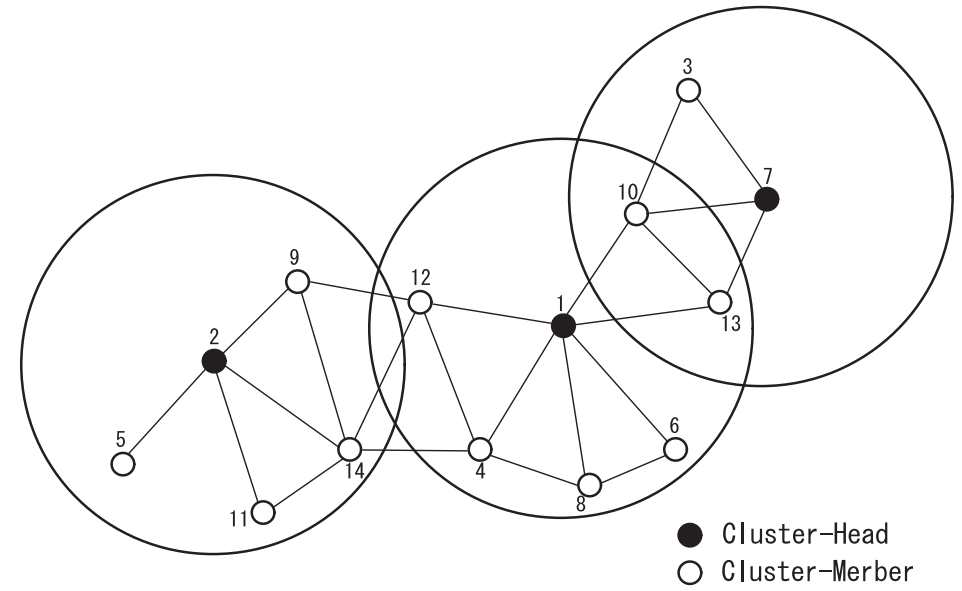

Fig. 5. $\mathrm{CH}$ selection process.

$$
\begin{aligned}
& \mu_{H g}(R P S)=g\left(R P S ; H g_{0}, H g_{1}, H g_{w 0}, H g_{w 1}\right) ; \\
& \mu_{F w}(D 3 N)=g\left(D 3 N ; F w_{0}, F w_{1}, F w_{w 0}, F w_{w 1}\right) ; \\
& \mu_{M e}(D 3 N)=f\left(D 3 N ; M e_{0}, M e_{w 0}, M e_{w 1}\right) ; \\
& \mu_{M n}(D 3 N)=g\left(D 3 N ; M n_{0}, M n_{1}, M n_{w 0}, M n_{w 1}\right) ; \\
& \mu_{N r}(D C C)=g\left(D C C ; N r_{0}, N r_{1}, N r_{w 0}, N r_{w 1}\right) ; \\
& \mu_{M o}(D C C)=f\left(D C C ; M o_{0}, M o_{w 0}, M o_{w 1}\right) ; \\
& \mu_{F r}(D C C)=g\left(D C C ; F r_{0}, F r_{1}, F r_{w 0}, F r_{w 1}\right) .
\end{aligned}
$$

The output linguistic parameter is the PCHS and we define the term set the same as FCHS System1: $\{\operatorname{Very}$ Weak $(V W)$, Weak $(W)$, Little Weak $(L W)$, Medium $(M D)$, Little Strong $(L S)$, Strong $(S)$, Very Strong $(V S)\}$.

The membership functions for the output linguistic parameter $P C H S$ are:

$$
\begin{aligned}
\mu_{V W}(P C H S) & =g\left(P C H S ; V W_{0}, V W_{1}, V W_{w 0}, V W_{w 1}\right) ; \\
\mu_{W}(P C H S) & =f\left(P C H S ; W_{0}, W_{w 0}, W_{w 1}\right) ; \\
\mu_{L W}(P C H S) & =f\left(P C H S ; L W_{0}, L W_{w 0}, L W_{w 1}\right) ; \\
\mu_{M D}(P C H S) & =f\left(P C H S ; M D_{0}, M D_{w 0}, M D_{w 1}\right) ; \\
\mu_{L S}(P C H S) & =f\left(P C H S ; L S_{0}, L S_{w 0}, L S_{w 1}\right) ; \\
\mu_{S}(P C H S) & =f\left(P C H S ; S_{0}, S_{w 0}, S_{w 1}\right) ; \\
\mu_{V S}(P C H S) & =g\left(P C H S ; V S_{0}, V S_{1}, V S_{w 0}, V S_{w 1}\right) .
\end{aligned}
$$

The fuzzy membership functions for input parameters are shown in Fig. 6. The FRB2 has 27 rules. 

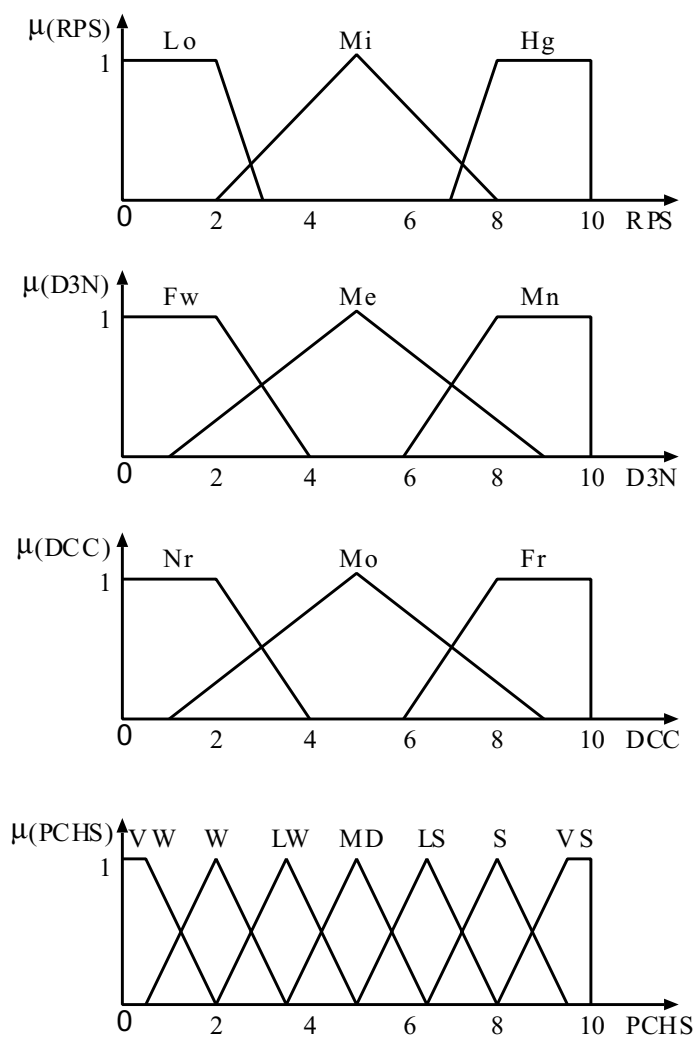

Fig. 6. Membership functions for FCHS system1.

\section{Simulation results}

In this section, we present the simulation results. We evaluate by computer simulations the performance of the previous system, FCHS System1 and FCHS System2.

In Fig. 7 is shown the performance evaluation of the previous system [20]. The graph in 3 dimensions shows the relation between the possibility of a sensor to be selected as a $\mathrm{CH}$ versus the distance and the remained sensor power. With the increase of the remained sensor power and the decrease of the distance between the sensor and sink, the possibility of a sensor to be selected as $\mathrm{CH}$ is increased. However, the graph surface is increased almost proportionally with the change of both parameters.

The performance evaluation of FCHS System1 is shown in Figs 8, 9 and 10. In Fig. 8 is shown the relation between the possibility of a sensor to be selected as a $\mathrm{CH}$ versus the distance and the remained sensor power. This is the same relation with the previous system. With the increase of the remained sensor power and the decrease of the distance between the sensor and the sink, the possibility of a sensor to be selected as $\mathrm{CH}$ is increased. However, the surface control of FCHS System1 is better than previous system, which results in a better $\mathrm{CH}$ selection and increase of the network lifetime. In Fig. 9 is shown the relation between the possibility a sensor to be selected as a $\mathrm{CH}$ versus the network traffic and the remained sensor power. The behavior of FCHS System1 for these parameters is almost the same with the performance in Fig. 8 .

In Fig. 10 is shown the relation between the possibility of a sensor to be selected as a $\mathrm{CH}$ versus the distance and the network traffic situation. With the increase of the network traffic and the increase of 


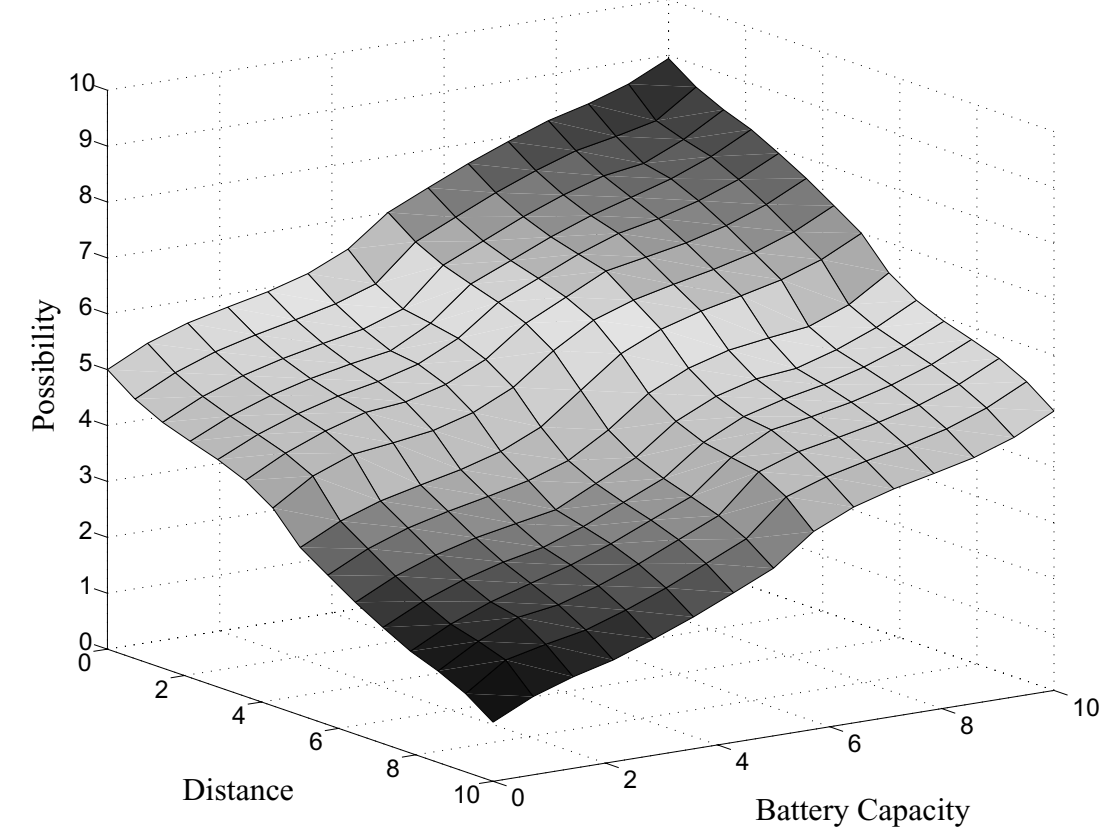

Fig. 7. Previous system simulation results.

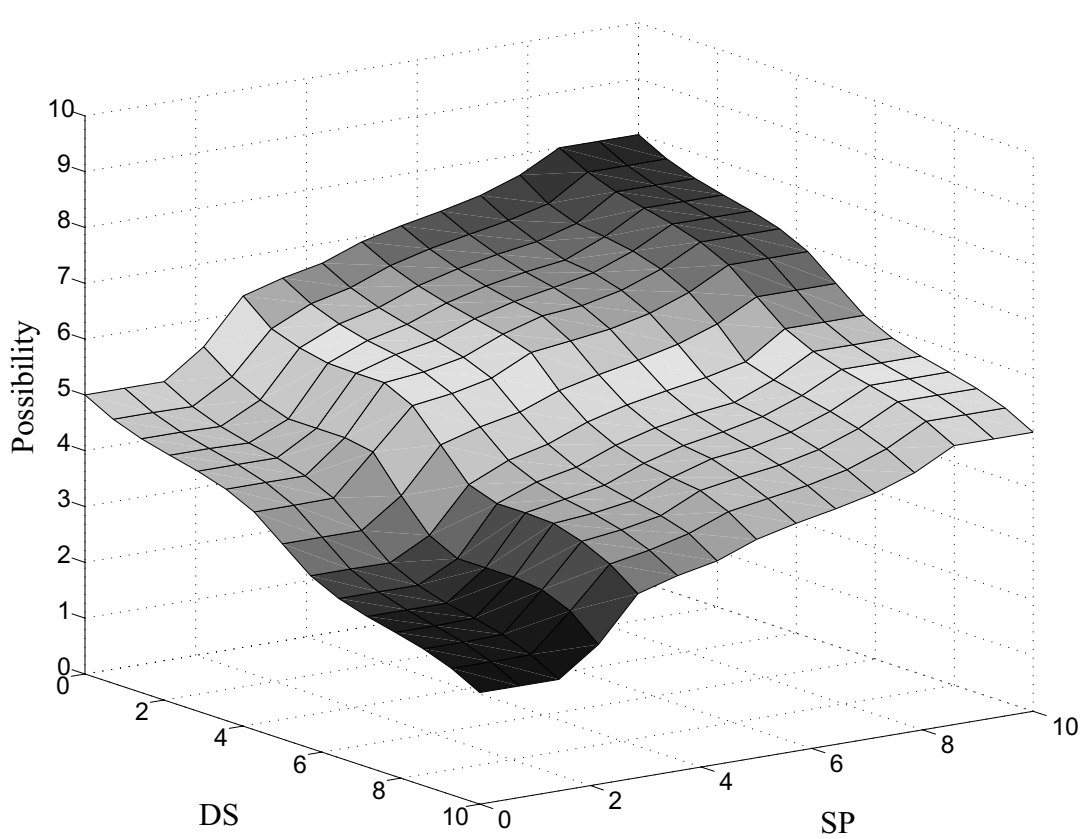

Fig. 8. FCHS System1 results (case 1).

the distance between the sensor and sink, the possibility of a sensor to be selected as $\mathrm{CH}$ is decreased. Different from the results in Figs 8 and 9, we see that the shape of the surface is changed proportionally with the changes of parameters. This shows, the remained battery power of a sensor is more important 


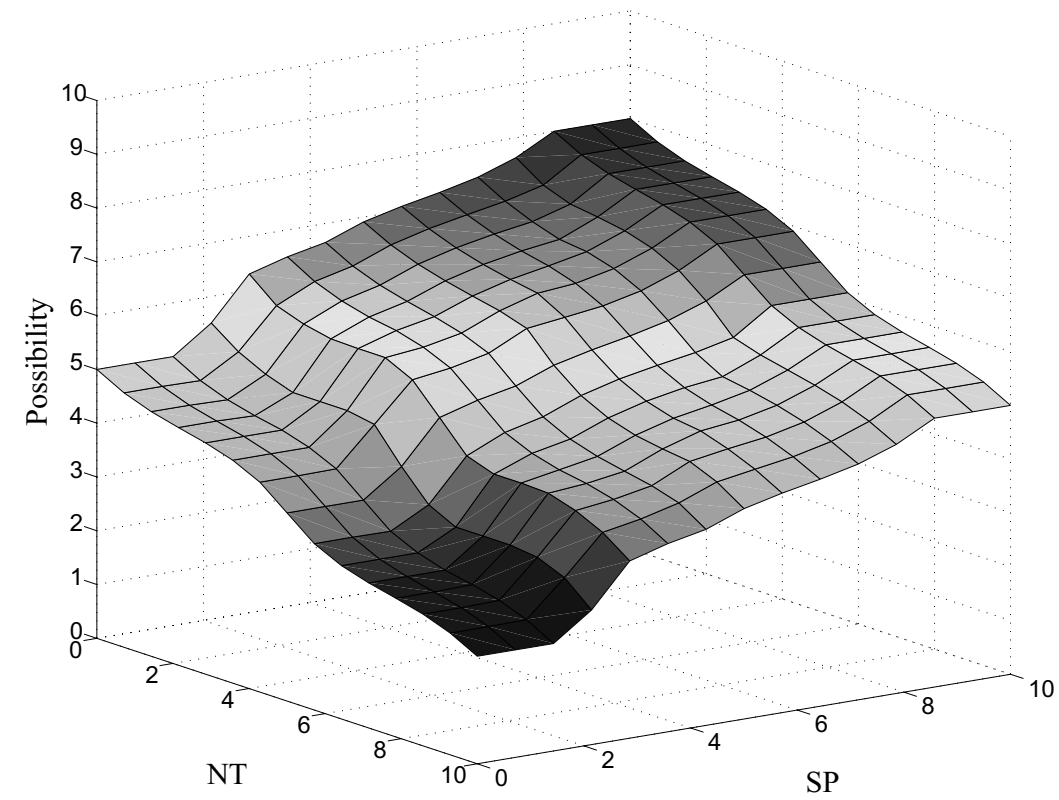

Fig. 9. FCHS System1 results (case 2).

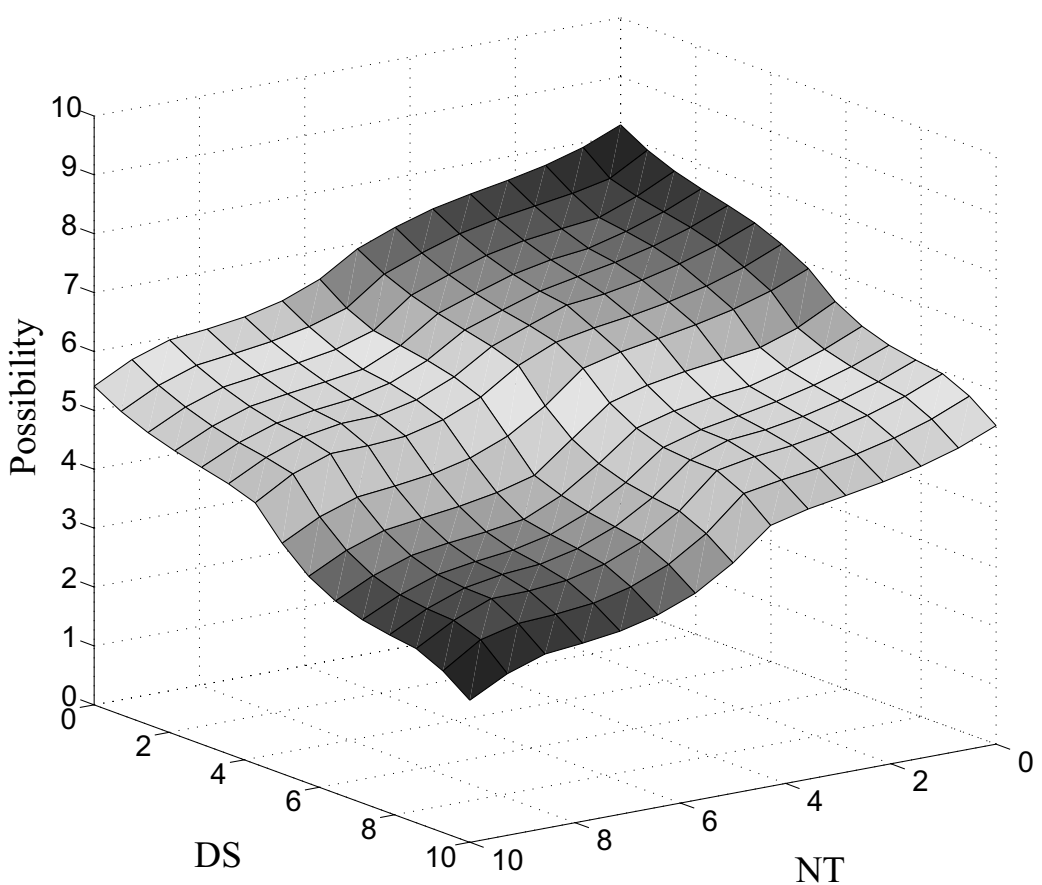

Fig. 10. FCHS System1 results (case 3).

for the selection of a $\mathrm{CH}$ than the traffic conditions.

We show the performance of FCHS System2 in Figs 11, 12 and 13. In Fig. 11 is shown the same relation with previous system and FCHS System1. The $\mathrm{CH}$ selection surface of proposed system is 


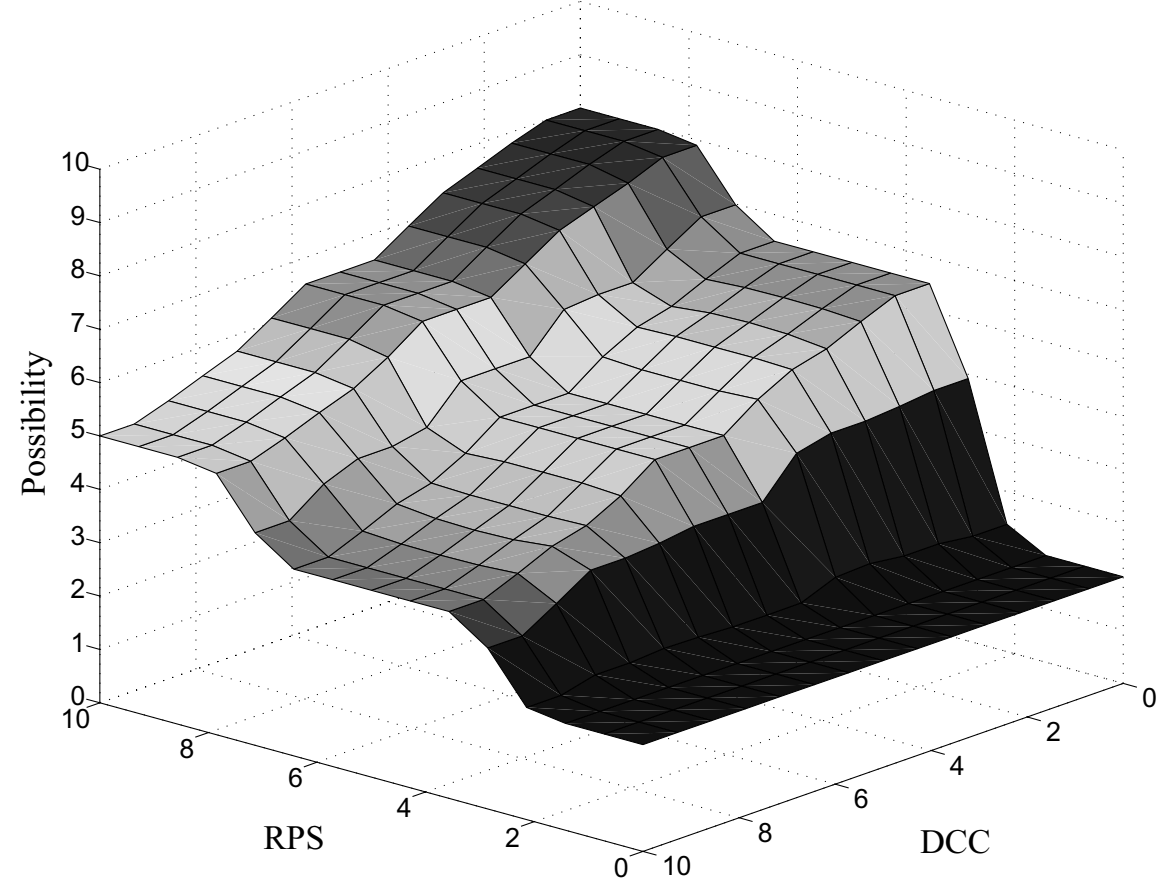

Fig. 11. FCHS System2 results (case 1).

better than the other systems. We clearly distinguish 3 zones. When, the RPS is less than 2 units the probability of a node to be selected as $\mathrm{CH}$ is very small. A middle zone (more than 2 units but less than 8 units), where the $\mathrm{CH}$ selection possibility increases slowly with increase of the RPS and decrease of DCC. A third zone (more than 8 units), where the possibility of a node to be $\mathrm{CH}$ is high. In this way, the proposed system can control better the RPS, which results in the increase of the network lifetime.

In Fig. 12 is shown the relation between the possibility of a sensor to be selected as a $\mathrm{CH}$ versus the D3N and RPS. Also, here we see 3 zones. When the RPS is low and the number of neighbor nodes (D3N) is high, the possibility that a node to be selected as CH is low. When the RPS is middle and the D3N increases, the possibility that a node to be selected as CH is increased. When the RPS is high and the $\mathrm{D} 3 \mathrm{~N}$ is high, the possibility that a node to be selected as $\mathrm{CH}$ is high.

In Fig. 13, we show the relation between possibility of a sensor to be selected as a $\mathrm{CH}$ versus the D3N and DCC. With the increase of D3N and the decrease of DCC, the possibility of a sensor to be selected as $\mathrm{CH}$ is increased. Different from the results in Figs 11 and 12, we see that the shape of the surface is changed proportionally with the changes of parameters. This shows that the RPS and 3DN parameters are more important for the selection of a $\mathrm{CH}$ than the DCC.

In Fig. 14, we show the comparison performance of the previous system, FCHS System1 and FCHS System2. When, the remaining battery power is more than 6 units, the previous system and FCHS System 1 have almost the same behavior. When, the remaining battery power is between 2 and 6 units, FCHS System1 has better behavior than the previous system. However, when the remaining battery power is less than 1 unit, the probability of $\mathrm{CH}$ selection of FCHS System 1 is higher than the previous system. The performance of FCHS System 2 is better than the previous system and FCHS System1. When the remaining battery power is less than 2 units, the probability of the sensor to be selected as $\mathrm{CH}$ in FCHS System2 is very small. When the remaining battery power is between 3 and 7 units, the 


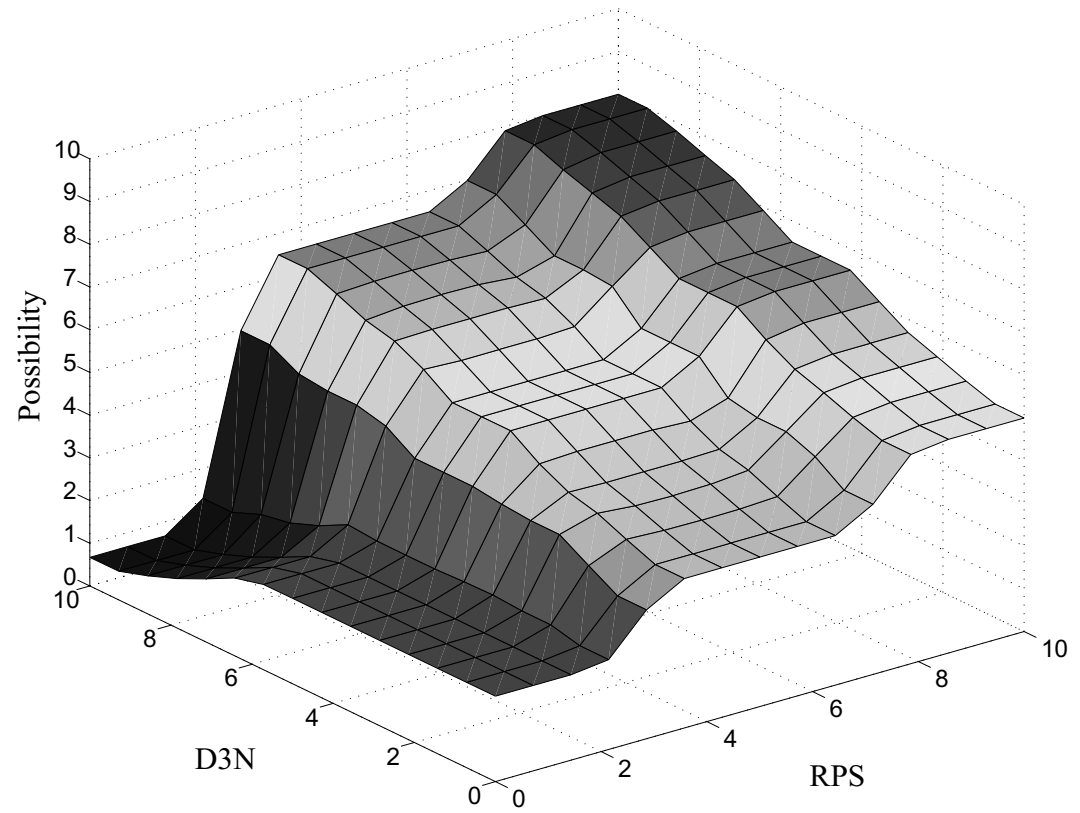

Fig. 12. FCHS System2 results (case 2).

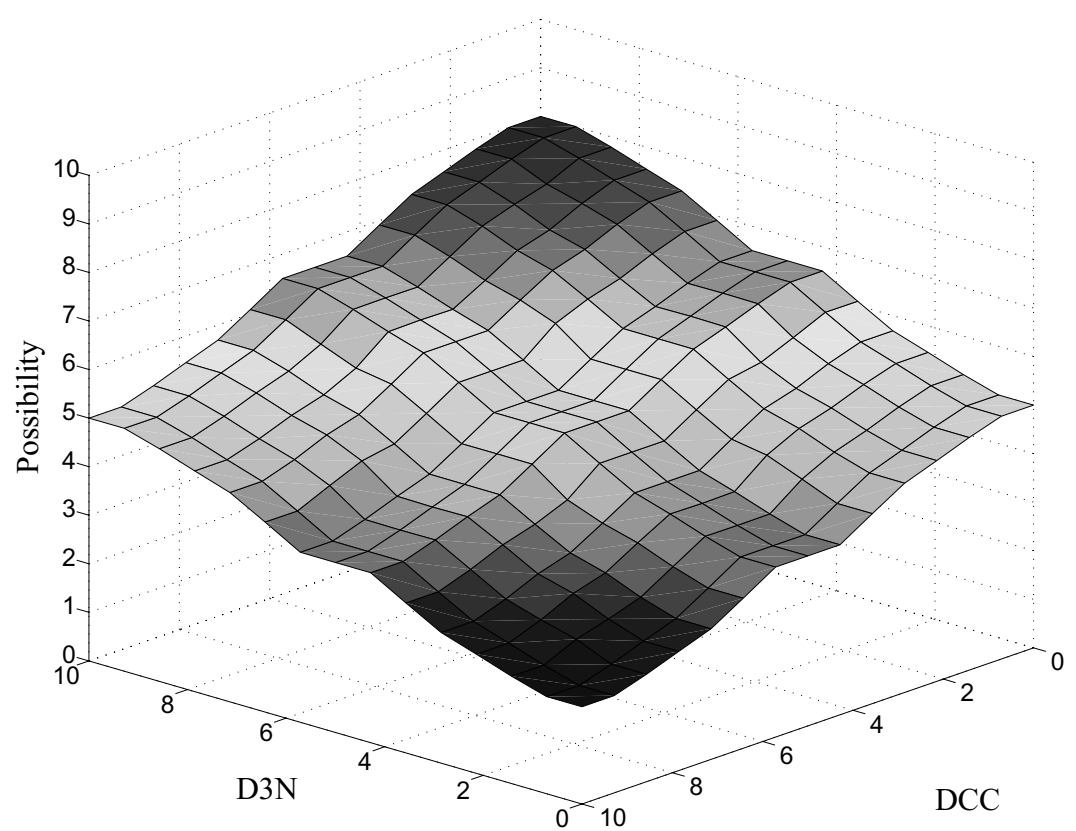

Fig. 13. FCHS System2 results (case 3).

possibility of $\mathrm{CH}$ selection is almost the same. For remaining battery power is more than 7 units, FCHS System 2 has almost the same behavior with other systems. In this way, FCHS System 2 can control better the energy of the sensor and thus increase the overall network lifetime. 


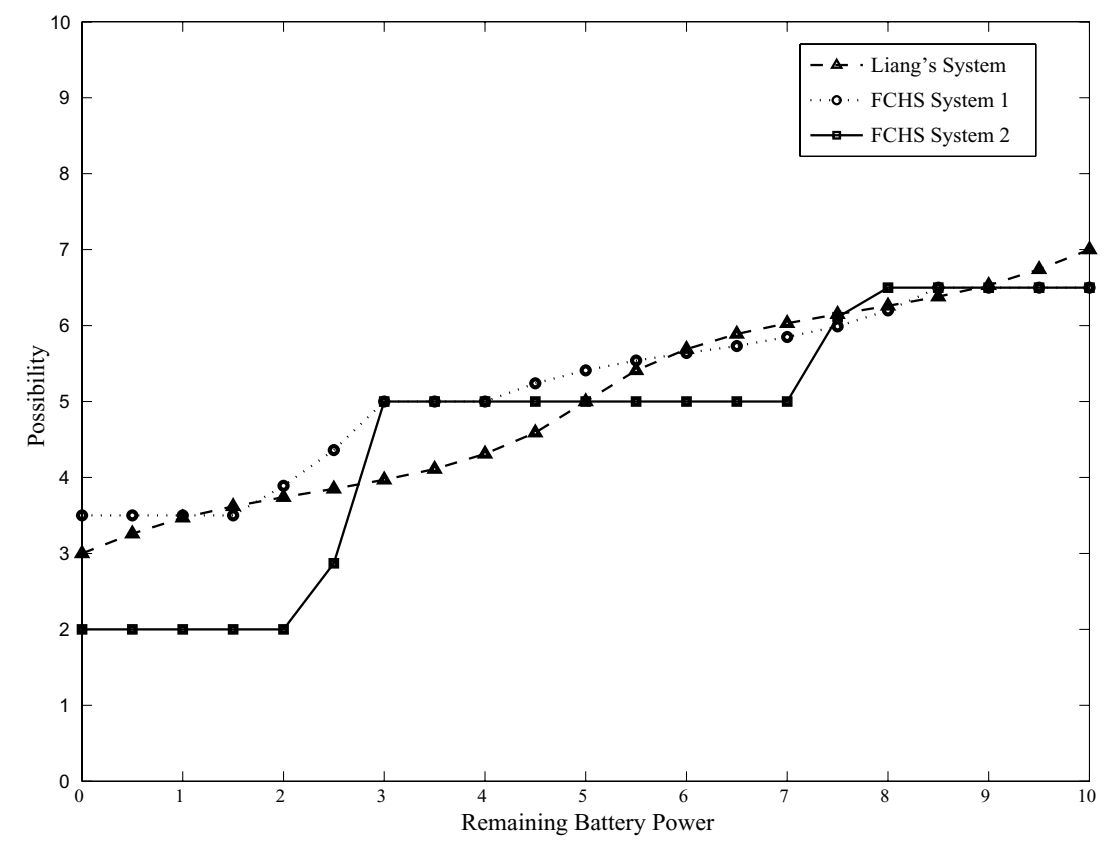

Fig. 14. Comparison between different systems.

\section{Conclusions}

The power conservation in WSN is a very important issue. Conserving power prolongs the lifetime of a node and also the lifetime of the whole network. Clustering is one of the energy-efficient techniques for extending the lifetime of a sensor network. Clustering techniques organize the nodes into clusters where some nodes work as $\mathrm{CHs}$ and collect the data from other nodes in the clusters. However, $\mathrm{CH}$ selection is very difficult when many parameters are used for making the decision.

In this paper, we proposed two $\mathrm{CH}$ selection systems based on FL. We evaluated their performance by computer simulations. The FCHS System 2 shows better performance than the previous system and FCHS System1. We found that, the remained energy of the sensor and the number of neighbor nodes are more important parameters for $\mathrm{CH}$ selection than the distance of the node from the sink.

We are working now to implement the proposed system in NS-2 simulator in order to compare the performance with other non fuzzy-based approaches such as LEACH. In the future, we want to extend our work for large-scale WSNs.

\section{Acknowledgment}

The authors would like to thank International Communications Foundation (ICF) of Japan and Japanese Society for the Promotion of Science (JSPS) for supporting this work.

\section{References}

[1] I.F. Akyildiz, W. Su, Y. Sankarasubramaniam and E. Cayirci, Wireless Sensor Networks: A Survey, Computer Networks 38(4) (2002), 393-442. 
[2] I.F. Akyildiz and I.H. Kasimoglu, Wireless Sensor and Actor Networks: Research Challenges, Ad Hoc Networks 2(4) (2004), 351-367.

[3] J.N. Al-Karaki and A.E. Kamal, Routing Techniques in Wireless Sensor Networks: A Survey, IEEE Wireless Communication 11(6) (2004), 6-28.

[4] A.D. Amis, R. Prakash, T.H.P. Vuong and D.T. Huynh, Max-Min D-cluster Formation in Wireless Ad Hoc Networks, Proc of IEEE INFOCOM-2000 (2000), 32-41.

[5] J. Anno, L. Barolli, F. Xhafa and A. Durresi, A Cluster Head Selection Method for Wireless Sensor Networks Based on Fuzzy Logic, Proc. of IEEE TENCON-2007 (2007), CD-ROM 4 pages.

[6] L. Barolli, A. Koyama, T. Suganuma and N. Shiratori, GAMAN: A GA Based QoS Routing Method for Mobile Ad-hoc Networks, Journal of Interconnection Networks (JOIN) 4(3) (2003) 251-270.

[7] L. Barolli, An Intelligent Call Admission Control System for Wireless Cellular Networks Based on Fuzzy Logic, Journal of Mobile Multimedia (JMM) 3(4) (2007), 331-346.

[8] L. Barolli, A Speed-aware Handover System for Wireless Cellular Networks Based on Fuzzy Logic, Mobile Information Systems 4(1) (2008), 1-12.

[9] S. Banerjee and S. Khuller, A Clustering Scheme for Hierarchical Control in Multi-hop Wireless Networks, Proc of IEEE INFOCOM-2001 (2001), 1028-1037.

[10] S. Basagni, Distributed Clustering for Ad Hoc Networks, International Symposium of Parallel Architectures, Algorithms and Networks (I-SPAN'99) (1999), 310-315.

[11] M. Chatterjee, S.K. Das and D. Turgut, WCA: A Weighted Clustering Algorithm for Mobile Ad Hoc Networks, Journal of Cluster Computing 5(2) (2002), 193-204.

[12] W.P. Chen, J.C. How and L. Sha, Dynamic Clustering for Acoustic Target Tracking in Wireless Sensor Networks, IEEE Trans on Mobile Computing 3(3) (2004), 258-271.

[13] H. Chan and A. Perrig, ACE: An Emergent Algorithm for Highly Uniform Cluster Formation, Proc of European Workshop on Wireless Sensor Networks (EWSN-2004) (2004), 154-171.

[14] P.M.L. Chan, R.E. Sheriff, Y. Hu, P. Conforto and C. Tocci, Mobility Management Incorporating Fuzzy Logic for a Heterogeneous IP Environment, IEEE Communications Magazine 39(12) (2001), 42-51.

[15] G. De Marco, T. Yang, M. Ikeda, L. Barolli, Performance Evaluation of Wireless Sensor Networks for Event-detection with Shadowing-induced Radio Irregularities, Mobile Information Systems 3(3-4) (2007), 251-266.

[16] S. Giordano and C. Rosenberg, Topics in Ad Hoc and Sensor Networks, Guest Editorial, IEEE Communication Magazine 44(4) (2006), 97.

[17] W.B. Heinzelman, A.P. Chandrakasan and H. Balakrishnan, An Application-specific Protocol Architecture for Wireless Microsensor Networks, IEEE Transactions on Wireless Communications 1(4) (2004), 660-670.

[18] W.R. Heinzelman, A. Chandrakasan and H. Balakrishnan, Energy-efficient Communication Protocol for Wireless Microsensor Networks, Proc of the 33rd Annual Hawaii International Conference on System Sciences (HICSS) (2000), 3005-3014.

[19] S. Lindsey, C. Raghavendra and K. M. Sivalingam, Data Gathering Algorithms in Sensor Networks Using Energy Metrics, IEEE Transactions on Parallel and Distributed Systems 13(9) (2002), 924-935.

[20] Q. Liang, A Design Methodology for Wireless Personal Area Networks with Power Efficiency, Proc of the Wireless Communications and Networking (WCNC) 3 (2003), 1475-1480.

[21] J.M. Mendel, Fuzzy Logic Systems for Engineering: A Tutorial, Proc of the IEEE 83(3) (1995), 345-377.

[22] T. Yang, G. De Marco, M. Ikeda, L. Barolli, Impact of Radio Randomness on Performances of Lattice Wireless Sensors Networks Based on Event-reliability Concept, Mobile Information Systems 2(4) (2006), 211-227.

Junpei Anno received BE degree from Fukuoka Institute of Technology, Japan in 2007. Presently, he is a MS student at Graduate School of Engineering, Fukuoka Institute of Technology. His research interests include wireless communications and networking, fuzzy logic and call admission control. is a student member of IEICE.

Leonard Barolli received BE and PhD degrees from Tirana University and Yamagata University in 1989 and 1997, respectively. From April 1997 to March 1999, he was a JSPS Post Doctor Fellow Researcher at Department of Electrical and Information Engineering, Yamagata University. From April 1999 to March 2002, he worked as a Research Associate at the Department of Public Policy and Social Studies, Yamagata University. From April 2002 to March 2003, he was an Assistant Professor at Department of Computer Science, Saitama Institute of Technology (SIT). From April 2003 to March 2005, he was an Associate Professor and presently is a Full Professor, at Department of Information and Communication Engineering, Fukuoka Institute of Technology (FIT). Dr. Barolli has published more than 250 papers in referred Journals and International Conference proceedings. He was an Editor of the IPSJ Journal and has served as a Guest Editor for many International Journals. Dr. Barolli has been a PC Member of many International Conferences and was the PC Chair of IEEE AINA-2004 and IEEE ICPADS-2005. He was General Co-Chair of IEEE AINA-2006 and IEEE AINA-2008, Workshops Chair of iiWAS-2006/MoMM-2006 and iiWAS-2007/MoMM-2007, 
Workshop Co-Chair of ARES-2007, ARES-2008 and IEEE AINA-2007. Presently, he is Workshop Chair o ARES-2009 and General Chair of CISIS-2009. Dr. Barolli is the Steering Committee Chair of CISIS International Conference and is serving as Steering Committee Member in many International Conferences. He is organizers of many International Workshops. His research interests include network traffic control, fuzzy control, genetic algorithms, agent-based systems, ad-hoc networks and sensor networks, Web-based applications, distance learning systems and P2P systems. He is a member of SOFT, IPSJ, and IEEE.

Arjan Durresi received his BE, MS and PhD at Polytechnic University of Tirana, Albania in 1986, 1990 and 1993, respectively. He holds a Post-Graduate Degree from Italian Telecommunication Institute, La Sapienza University, Italy in 1991. He also got another PhD degree at Tokyo Denki University, Japan in 2006. Presently, he is an Associate Professor of Computer Science at Indiana University Purdue University at Indianapolis, Indiana. In the recent past, he also held roles as Associate Professor at Louisiana State University, Research Scientist of Computer Science and Adjunct Assistant Professor of Electrical and Computer Engineering at The Ohio State University. Dr. Durresi serves as Area Editor of Ad Hoc Networks Journal. He is on Editorial Boards of many International Journals. He has been a Guest Editor for many Special Issues. He was Workshops Co-Chair for 21th IEEE International Conference on Advanced Information Networking and Applications AINA-2007. He was Program Co-Chair of the AINA-2006. He is organizing many International Workshops. He has authored more than 50 papers in Journals and more than 80 in Conference Proceedings. Dr. Durresi's research interest include: network architectures, heterogeneous wireless ad hoc and sensor networks, security, QoS routing protocols, traffic management, grid computing, optical and satellite networks, multimedia networking, and bioinformatics.

Fatos Xhafa holds a PhD in Computer Science (1998) from the Department of Languages and Informatics Systems, Polytechnic University of Catalonia, Barcelona, Spain. He was graduated in Mathematics from the Faculty of Natural Sciences (FNS), University of Tirana (UT), Albania, in 1988. During 1989-1993 he was an Assistant Professor at the Department of Applied Mathematics of the FNS, UT (Albania). He joined the Polytechnic University of Catalonia as a Visiting Professor at the Applied Mathematics Department I, and later he entered the Department of Languages and Informatics Systems (LSI) as a PhD student and in 1996 as an Assistant Professor. Currently, he is an Associate Professor at the Department of Languages and Informatics Systems and member of the ALBCOM Research Group He is also a collaborator of the Open University of Catalonia (Barcelona, Spain). His current research interests include parallel and distributed algorithms, combinatorial optimization and approximation, meta-heuristics for complex problems, distributed programming, Grid and P2P computing. His research is supported by several research projects from Spain, European Union and NSF/USA. He collaborates with different international research groups.He has published in leading international journals and conferences and has served in the Organizing Committees of many conferences and workshops. He is currently the Organizing Chair of ARES-2008, PC Chair of CISIS-2008, Workshops Co-chair of CISIS-2008 and General Co-chair of HIS-2008 conferences. He is also member of editorial board of several international journals.

Akio Koyama received his BE and PhD degrees from Yamagata University in 1987 and 1998, respectively. From April 1999 to March 2002, he was an Assistant Professor at the Faculty of Computer Science and Engineering, the University of Aizu. From April 2002, he was an Associate Professor and presently is a Full Professor at the Faculty of Engineering, Yamagata University. Dr. Koyama has published about 100 papers in refereed journals and international conference proceedings. Dr. Koyama has been a PC member of many international conferences. He was the General Co-chair of MNSA-2005 and he is the Workshop Co-Chair of IEEE AINA-2008. His research interests include medical and care communication systems, routing protocols, high-speed network protocols, agent-based systems, distance learning systems and mobile communication systems. He is a member of the IEEE Computer Society, IPSJ and IEICE. 

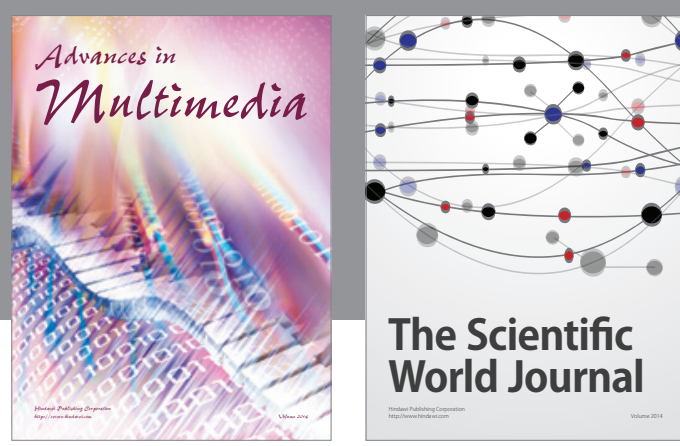

The Scientific World Journal
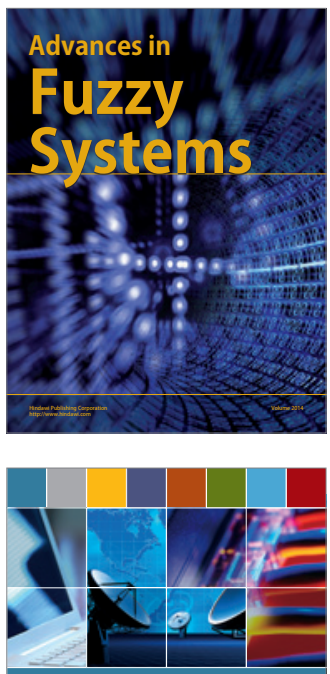

Computer Networks and Communications
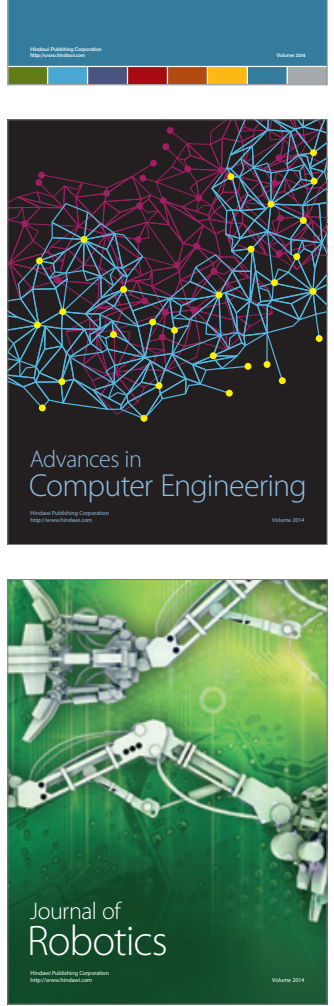
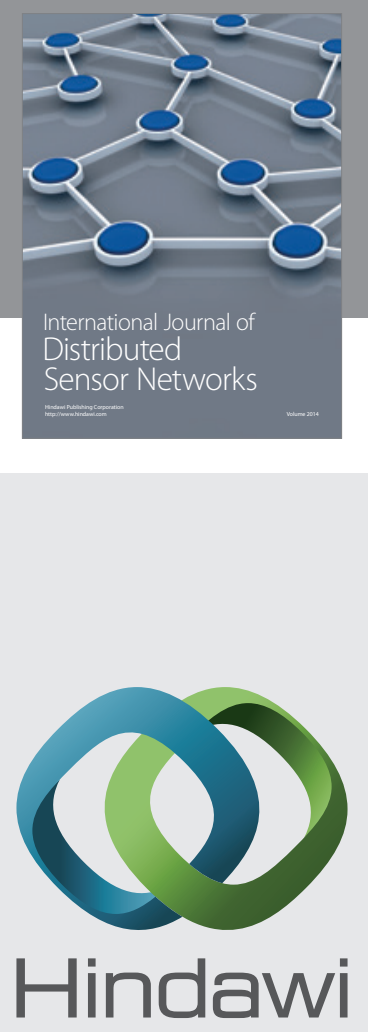

Submit your manuscripts at

http://www.hindawi.com
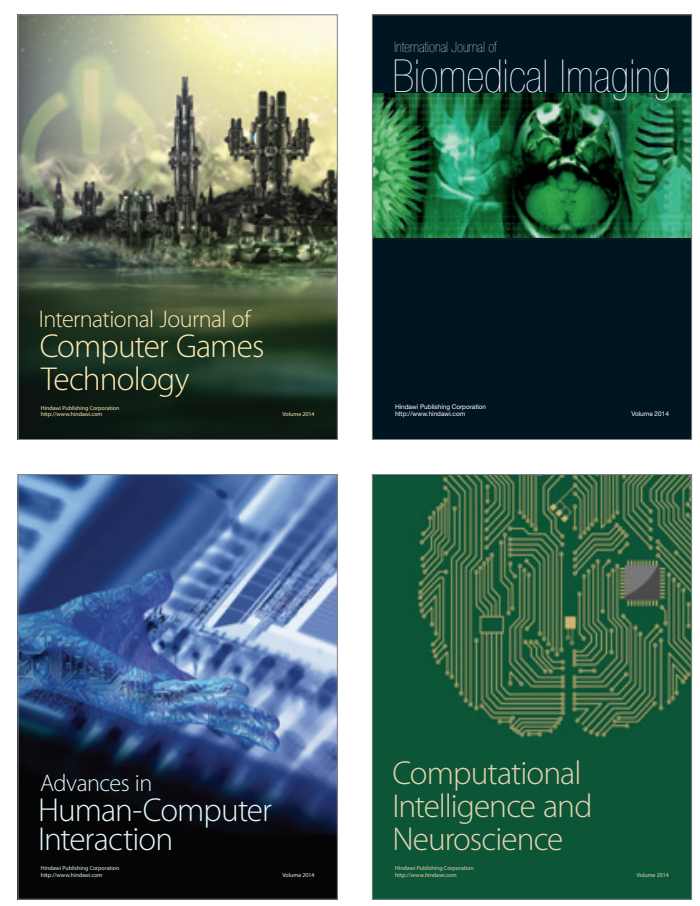
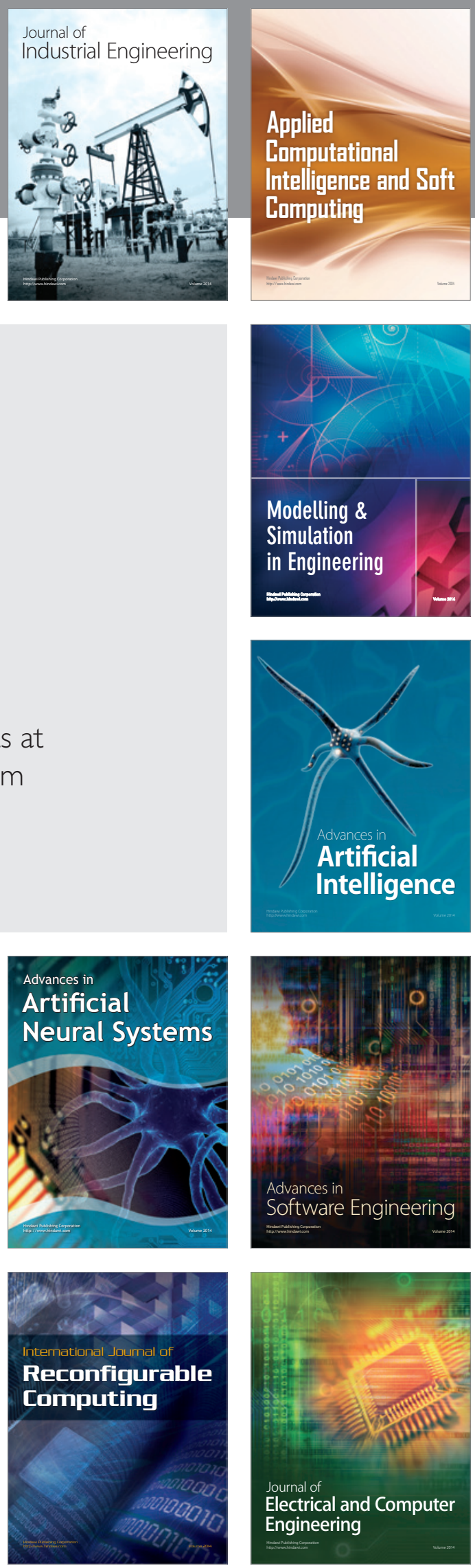